\title{
Altered Epithelial-mesenchymal Plasticity as a Result of Ovol2 Deletion Minimally Impacts the Self-renewal of Adult Mammary Basal Epithelial Cells
}

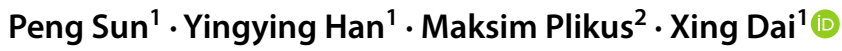

Received: 6 August 2021 / Accepted: 20 December 2021 / Published online: 4 January 2022

(c) The Author(s) 2022

\begin{abstract}
Stem-cell containing mammary basal epithelial cells exist in a quasi-mesenchymal transcriptional state characterized by simultaneous expression of typical epithelial genes and typical mesenchymal genes. Whether robust maintenance of such a transcriptional state is required for adult basal stem cells to fuel self-renewal and regeneration remains unclear. In this work, we utilized SMA-CreER to direct efficient basal cell-specific deletion of Ovol2, which encodes a transcription factor that inhibits epithelial-to-mesenchymal transition (EMT), in adult mammary gland. We identified a basal cell-intrinsic role of $\mathrm{Ovol} 2$ in promoting epithelial, and suppressing mesenchymal, molecular traits. Interestingly, Ovol2-deficient basal cells display minimal perturbations in their ability to support tissue homeostasis, colony formation, and transplant outgrowth. These findings underscore the ability of adult mammary basal cells to tolerate molecular perturbations associated with altered epithelia-mesenchymal plasticity without drastically compromising their self-renewal potential.
\end{abstract}

Keywords Mammary gland $\cdot$ Basal cell $\cdot$ Stem cell $\cdot$ Ovol2 $\cdot$ Epithelial-to-mesenchymal transition $\cdot$ Epithelialmesenchymal plasticity

\section{Introduction}

The mouse mammary gland is a dynamic and regenerative organ that undergoes most of its development after birth, with dramatic structural and/or functional changes occurring during puberty, estrus cycle, pregnancy, lactation, and involution $[1,2]$. Under the regulation of hormones and local growth factors, stem/progenitor cells in the mammary epithelium self-renew, proliferate, and differentiate to drive the growth, remodeling, and regeneration of the bi-lineage epithelial network composed of an outer basal layer and an inner luminal layer [3]. The basal cells differentiate into myoepithelial cells that

Xing Dai

xdai@uci.edu

1 Department of Biological Chemistry, School of Medicine, University of California, CA, Irvine 92697, USA

2 Department of Developmental and Cell Biology, University of California, Irvine, CA 92697, USA exhibit both epithelial and smooth muscle characteristics, and they contain unipotent and multi/bi-potent stem cell subsets that fuel morphogenesis and regeneration [3-10].

Epithelial-to-mesenchymal transition (EMT) is a form of cellular plasticity where epithelial cells lose or attenuate epithelial traits and gain mesenchymal traits [11-16]. It has become increasingly clear that EMT in vivo is not a binary process, but rather encompasses a heterogeneous spectrum of diverse intermediate or hybrid cellular states that exist between epithelial and mesenchymal phenotypes. Interestingly, mammary basal/myoepithelial cells express both epithelial and mesenchymal genes, and are in a "quasi-mesenchymal" transcriptional state that resembles intermediate cell states within the epithelialmesenchymal spectrum [17-20]. In breast cancer cells, expression of core EMT-inducing transcription factors is linked to the acquisition of stem cell traits [21-26]. How the "quasi-mesenchymal" transcriptional state is maintained in adult mammary tissue basal cells and whether aberrant epithelial-mesenchymal plasticity (EMP) alters stemness remain to be fully understood. 
We previously reported that deletion of EMT-suppressive transcription factor $O$ vol 2 in the entire mammary epithelium halts ductal morphogenesis during pubertal development $[27,28]$. Here we use SMA-CreER to drive the deletion of $O$ vol 2 specifically in adult mammary basal cells, and find that this leads to significantly enhanced EMT-like molecular features. However, the Ovol2-deficient basal cells are not outcompeted by Ovol2-intact basal cells during tissue homeostasis, and are capable of regenerating new mammary trees upon transplantation and producing colonies in vitro. Interestingly, acute deletion of Ovol 2 in cultured basal cells results in increased colony formation. Together, these findings reveal a context-dependent role of Ovol2 in basal cell proliferation/self-renewal and the robustness of adult mammary basal cells in tolerating EMT-like gene expression perturbations.

\section{Results}

\section{SMA-CreER Directs Inducible, Efficient, and Specific Gene Deletion in Adult Mammary Basal Cells}

SMA-CreER has been used to lineage-trace mammary basal cells [7], but its utility in directing basal cell-specific gene deletion for functional studies has not been explored. Towards that end, we bred SMA-CreER mice with $t d T o$ mato mice, and treated the resulting SMA-CreER;tdTomato females with two doses of tamoxifen in adulthood (Fig. 1a). Two weeks after treatment, we detected robust tdTomato fluorescence in mammary ducts and blood vessels using wholemount microscopy (Fig. 1b, middle). Immunostaining with K14 antibody to mark basal cells revealed nuclear tdTomato expression in the basal layer (Fig. 1b, right). Flow cytometry showed that $>80 \%$ of the basal cells express tdTomato,
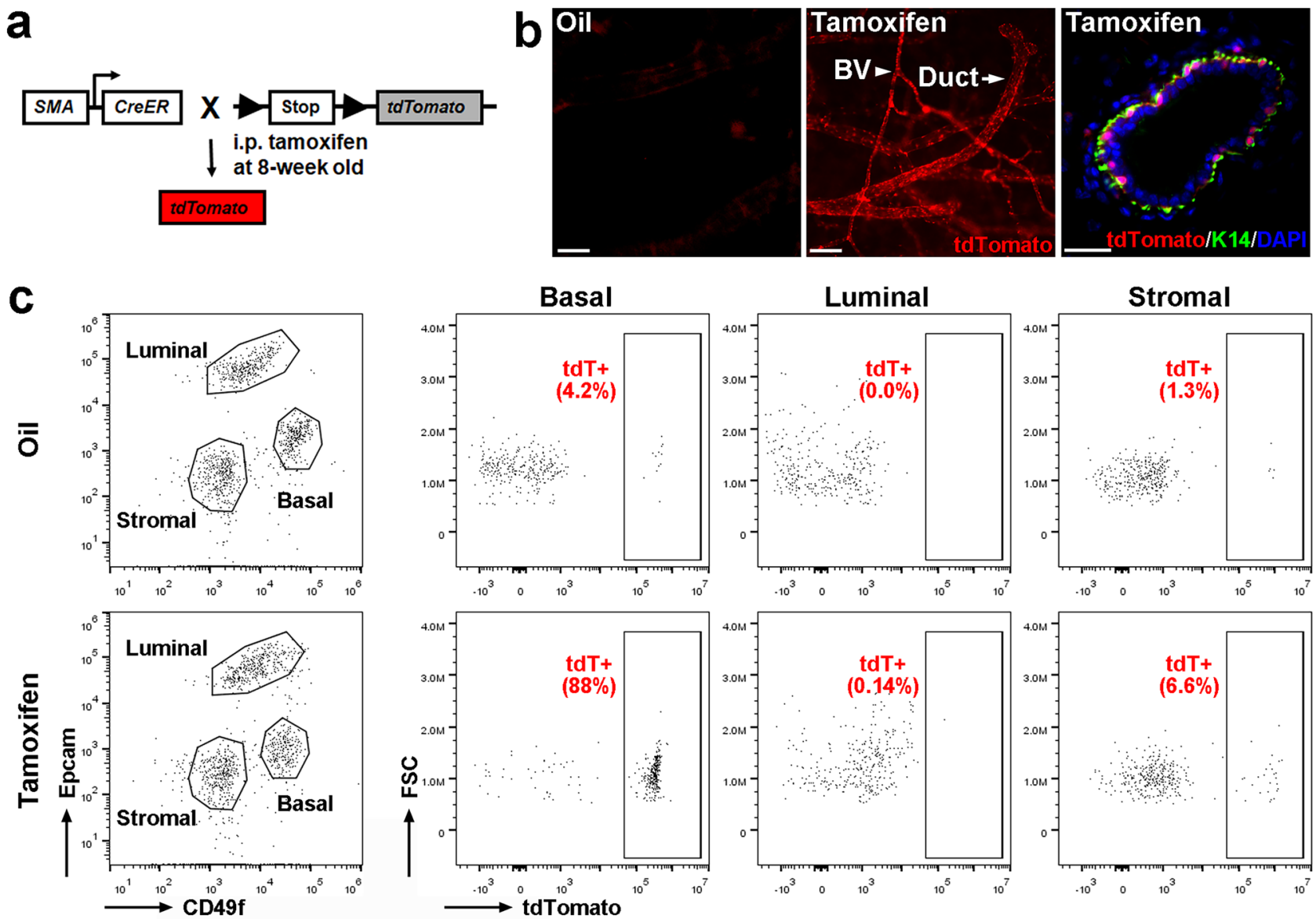

Fig. 1 SMA-CreER directs basal cell-specific recombination in adult mammary gland. a, Experimental design. b, Microscopic analysis of tdTomato fluorescence in whole-mount preparation (middle) and section (right) of mammary glands from SMA-CreER;tdTomato females at 14 days after tamoxifen injection. Oil was used as a negative con- trol (left). BV, blood vessel. Scale bar: left and middle, $100 \mu \mathrm{m}$; right, $25 \mu \mathrm{m}$. c, Representative flow cytometry profiles of immunostained cell lineage surface markers (left) and tdTomato (tdT) fluorescence (right). FSC, forward scatter. Mammary glands were harvested at 14 days after injection 
whereas less than $1 \%$ of the luminal cells and $\sim 7 \%$ of the stromal cells are tdTomato ${ }^{+}$(Fig. 1c). These data show that $S M A-C r e E R$ is a useful tool for adult mammary basal celltargeted gene deletion.

\section{Ovol2 Acts Cell-autonomously Within the Basal Layer to Promote Epithelial, and Suppress EMT-associated, Gene Expression}

Towards assessing the function of Ovol2 specifically in the mammary basal layer, we used SMA-CreER mice to inducibly delete Ovol2 in adulthood. SMA-CreER; Ovoll ${ }^{f /-}$ (inducible, basal cell-specific knockout or iBSKO) mice were generated and treated with tamoxifen at eight weeks of age along with control littermates. With time, the basal cells in iBSKO mice exhibited visible reduction in surface level of epithelial marker protein Epcam (Fig. 2; Online Resource 1a). Importantly, Epcam ${ }^{\text {low }}$ basal cells were detected only with tamoxifen injection, but not with oil injection (Online Resource 1b), indicating that their emergence is dependent on Ovol2 deletion. The relative
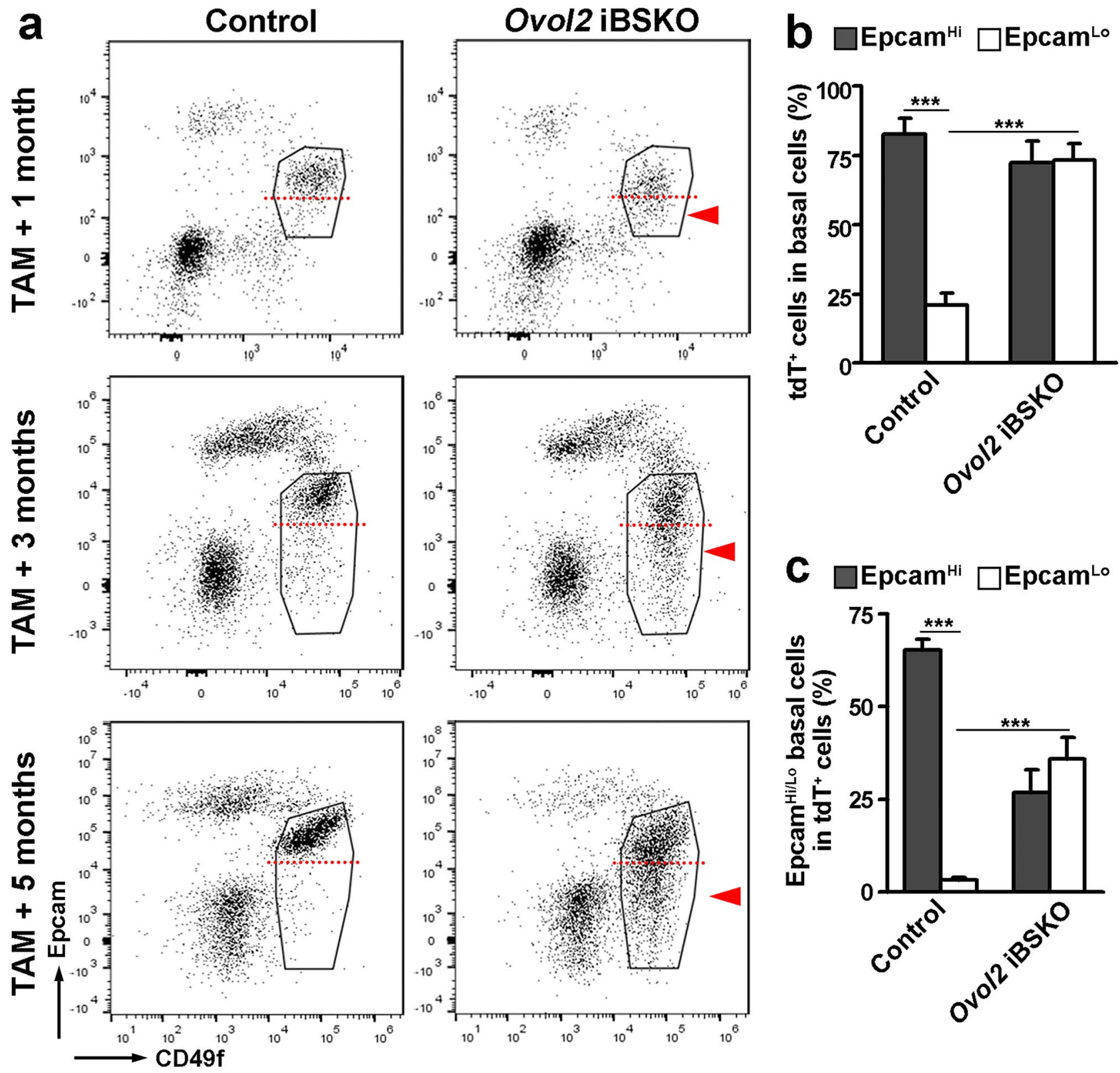

Fig. 2 Emergence of Epcam ${ }^{\text {low }}$ basal cells in Ovol2 iBKSO mice. a, Representative flow cytometry profiles of mammary cells at the indicated time points after tamoxifen induction. Red arrowheads point to Epcam $^{\text {low }}$ basal cells, demarcated by dashed red lines from Epcam ${ }^{\text {high }}$ basal cells. b, Quantitative analysis of tdTomato-positive cells in
Epcam $^{\text {low }}$ and Epcam ${ }^{\text {high }}$ basal cells at three months after tamoxifen injection. c. Quantitative analysis of Epcam ${ }^{\text {low }}$ and Epcam ${ }^{\text {high }}$ cells in tdTomato-positive cells at three months after tamoxifen injection. $\mathrm{n}=5$ pairs of mice in (b) and (c). ${ }^{* * *} p<0.005$. tdT, tdTomato. Control (Ctrl) genotype: SMA-CreER;Ovol2 ${ }^{f /+}$;tdTomato 
frequencies of basal and luminal cells were not affected (Online Resource 1c).

To determine if basal-specific Ovol2 deletion affects EMT-associated gene expression, we FACS-sorted basal cells at three months after tamoxifen induction, and performed RT-qPCR analysis. Ovol2 iBKSO basal cells showed significantly increased expression of mesenchymal gene $\mathrm{Vim}$ (encoding vimentin), as well as of Zebl and Twist, which encode EMT-inducing transcription factors (Fig. 3a). In contrast, the expression of epithelial adhesion gene $C d h l$ was significantly decreased in Ovol2 iBKSO basal cells, whereas the expression of other EMT-inducing transcription factors Snail and Snai2 was not affected (Fig. 3a). Immunofluorescence also detected more prominent vimentin protein expression in the mammary basal, but not luminal, cells of iBSKO mice compared to control littermates (Fig. 3b). Together, these data show that $O v o l 2$ is required in the adult basal cells to promote the epithelial-associated, and suppress the EMT/ mesenchymal-associated, molecular traits, thereby maintaining a quasi-mesenchymal transcriptional state and keeping EMT-like changes in check.

\section{Basal Cell-specific Deletion of Ovol2 Does Not Impact Mammary Homeostasis}

To examine the functional impact of Ovol2 loss-induced alteration in basal EMP, mammary gland morphology of
Ovol2 iBSKO and control littermates was analyzed at one month and three months after induction of gene deletion. No significant difference was observed in either ductal length or branching complexity (Fig. 4a, b). iBSKO and control littermates were also bred at one month after tamoxifen induction, and their pups were found to gain weight similarly (Online Resource 2), implying that lactation may not be dramatically affected by $O$ vol2 deletion in the basal cells.

To ask if the residual Ovol2-intact basal cells in Ovol2 iBSKO mice preferentially contribute to mammary epithelial homeostasis, we performed flow cytometry to compare the percent of tdTomato ${ }^{+}$(i.e., Ovol2-deleted) basal cells in control and iBSKO mice that also contain the tdTomato allele at one month and three months after tamoxifen treatment. At both times, tdTomato ${ }^{+}$basal cells were present at comparable frequencies in mammary glands from control and iBSKO mice (Fig. 4c, d). Moreover, the percent of tdTomato ${ }^{-}$(i.e., Ovol2-intact) did not increase over time in iBSKO mice (Fig. 4c, d). These data argue against the possibility that residual Ovol2-intact basal cells outcompete Ovol2-deficient basal cells during adult tissue homeostasis.

\section{Ovol2 Expression in Basal Cells is Largely Dispensable for Self-renewal and Regeneration}

To examine whether Ovol2 loss from the basal cells impacts their stem cell function, we conducted transplantation

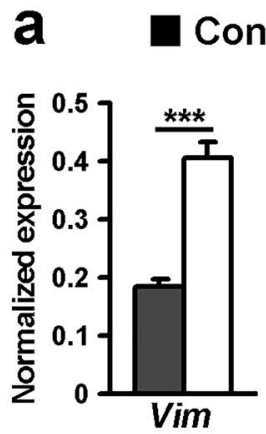

Control

Ovol2 iBSKO
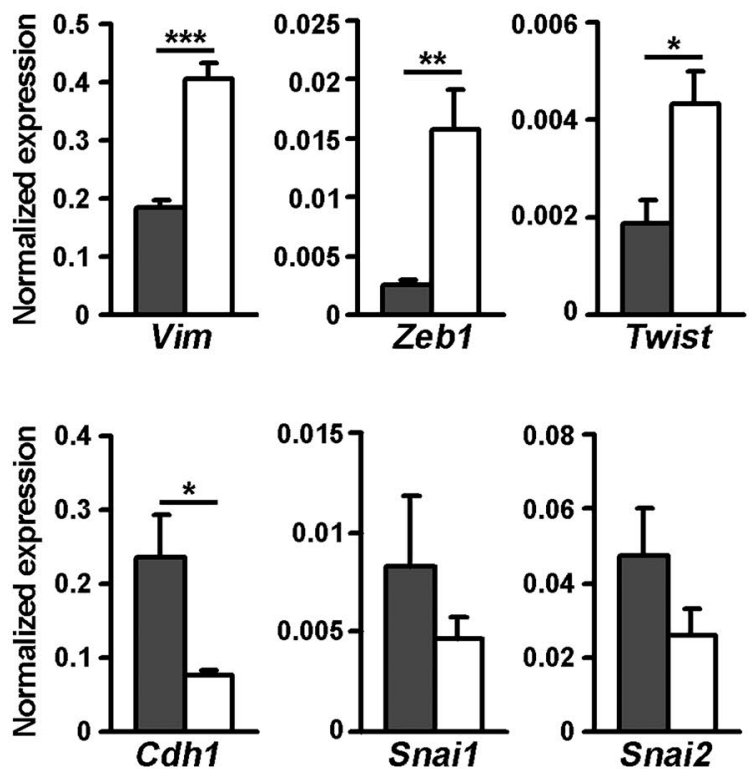

Fig. 3 Molecular changes in Ovol2 iBKSO basal cells suggests a partial epithelial-to-mesenchymal drift. a, RT-qPCR analysis of the indicated genes. $\mathrm{n}=4$ for Zeb1 and $\mathrm{n}=3$ for Vim, Twist, Cdh1, Snail and Snai2. *** $p<0.005$, ** $p<0.01$, * $p<0.05$. b, Immunofluorescence of vimentin protein. K14 and K8 antibodies stain basal cells
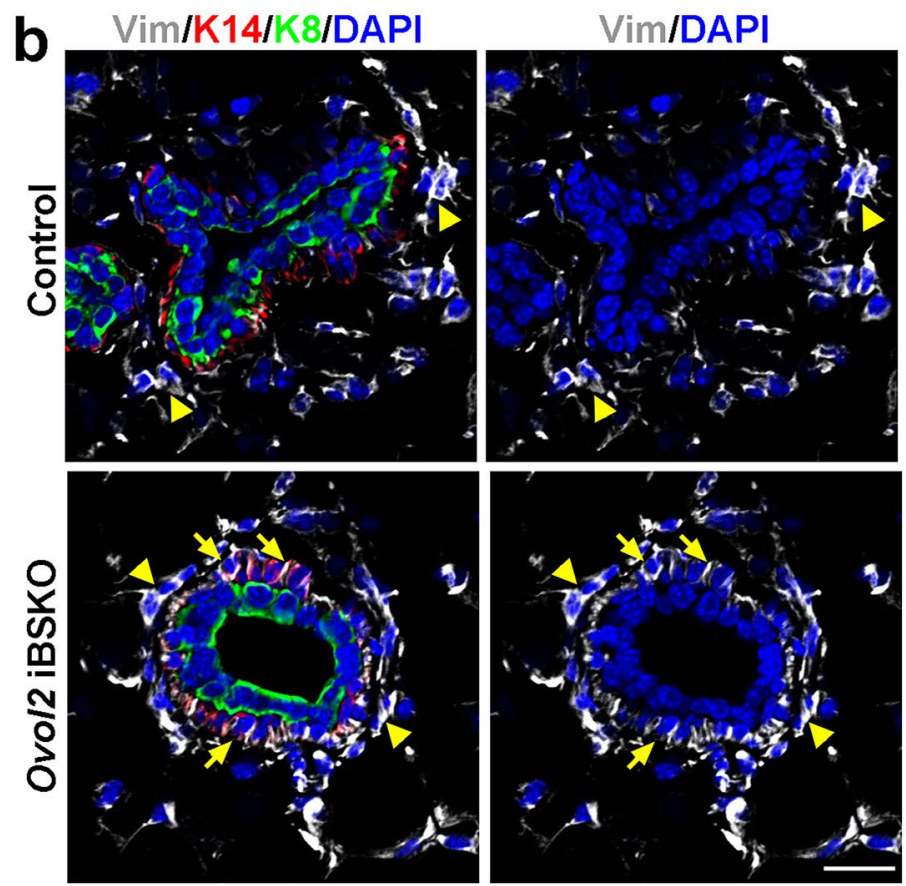

and luminal cells, respectively. DAPI stains the nuclei. Arrows and arrowheads point to vimentin-positive basal cells and stromal cells, respectively. Scale bar: $25 \mu \mathrm{m}$. Mammary glands were harvested at three months after tamoxifen injection. Control genotype: SMACreER;Ovol2 ${ }^{f /+} ;$ tdTomato 

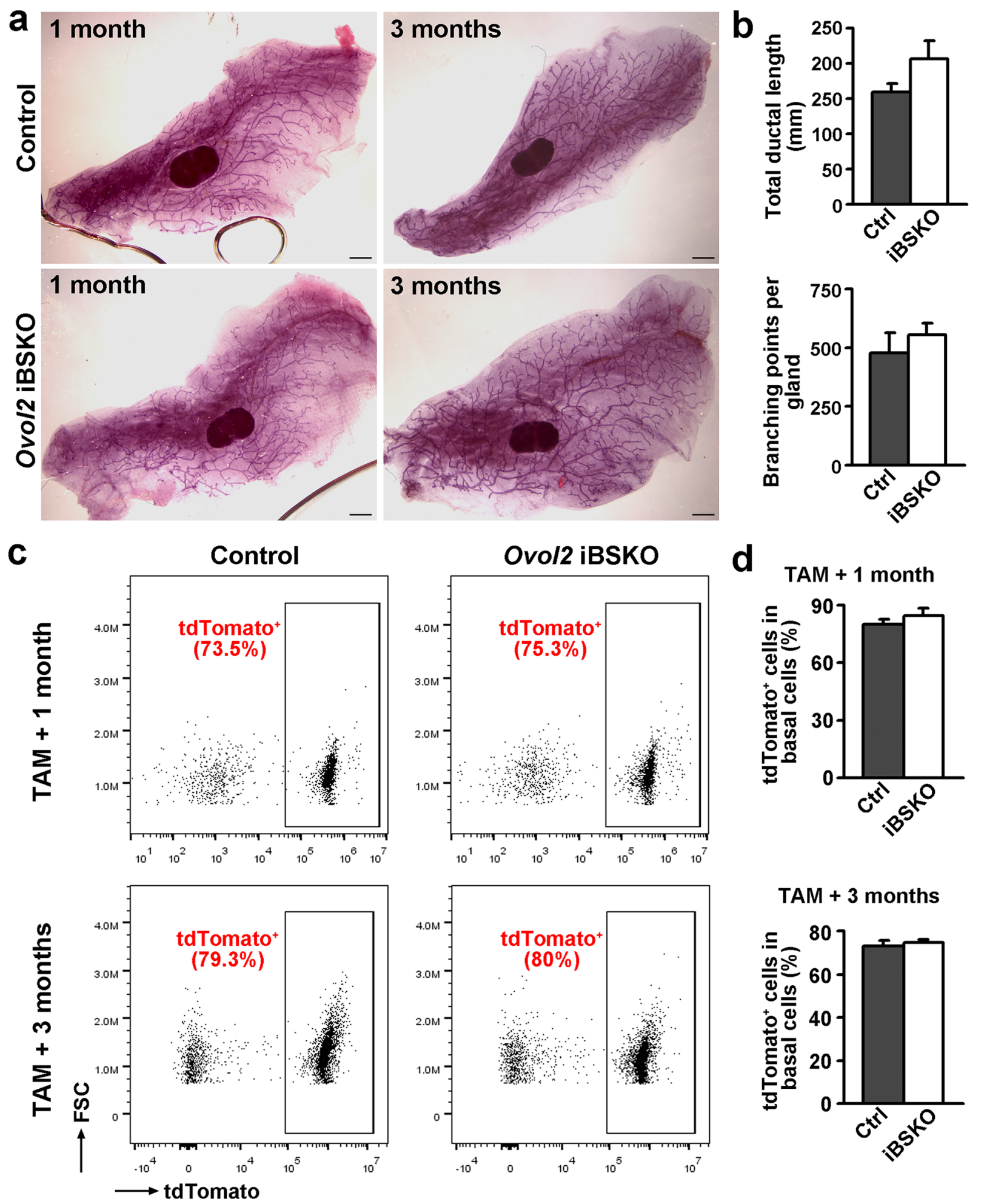

\section{TAM +3 months}

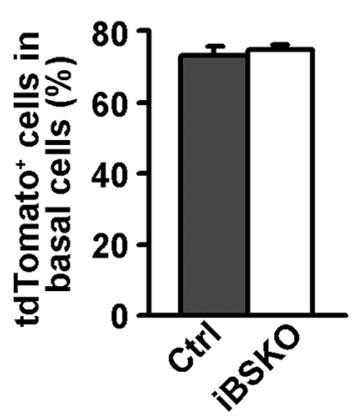

Fig. 4 Ovol2 loss from adult basal cells does not alter mammary gland homeostasis. a, Representative whole-mount carmine staining images of mammary glands from control and Ovol2 iBSKO littermates at the indicated times after tamoxifen injection. Scale bar: $1 \mathrm{~mm}$. b, Quantitative analysis of ductal length and branching at three months after tamoxifen injection. $\mathrm{n}=3$ pairs of mice. $\mathbf{c}$, Representative flow cytom- etry profiles to show frequency of tdTomato-positive cells in control and Ovol2 iBSKO basal cells at the indicated times after tamoxifen injection. d, Quantitative analysis of tdTomato-positive basal cells as in (c). $n=3$ pairs of mice for one month and $n=6$ pairs for three months of age. FSC, forward scatter. Control (Ctrl) genotype: SMACreER;Ovol2 ${ }^{\text {f/ }}$; tdTomato 
experiments by injecting FACS-sorted tdTomato ${ }^{+}$basal cells from Ovol2 iBSKO and control littermates into de-epithelialized fat pads of congenic 3-week-old host mice. Using serially decreasing numbers of basal cells, fat pad filling by Ovol2deficient basal cells was overall similar to that by control basal cells (Fig. 5a-c; Online Resource 3a). The overall take rate for all transplants analyzed was $85 \%$ (17/20) for control and $80 \%$ (16/20) for Ovol2-deficient basal cells (Fig. 5b). We also analyzed the epithelial structure of the transplants using H/E staining and immunofluorescence, which revealed largely normal ductal histology and expression of basal (K14) and luminal (K8) keratin markers (Fig. 5d, e). These results suggest that $\mathrm{Ovol} 2$ expression in adult basal cells is not essential for the self-renewal and regenerative potential of these cells.

To complement the in vivo findings above, we next performed ex vivo 3D-Matrigel colony formation assays by culturing sorted tdTomato ${ }^{+}$basal cells from $\mathrm{Ovol} 2$
iBSKO (enriched for Ovol2-deficient cells) and control littermates. Colonies formed by the Ovol2 iBSKO basal cells were similar in number and size to those formed by control cells both in initial plating and when the cells were serially passaged (Fig. 5f; Online Resource 3b). To ask whether Ovol2 may play a role in adult basal cell proliferation/self-renewal that only manifests under certain conditions, we sorted basal cells from adult mice containing floxed $O$ vol 2 alleles $\left(O v o l 2^{\mathrm{f} / \mathrm{f}}\right)$, infected them with Cre-expressing adenoviruses (Ade-Cre) to acutely delete Ovol2 [29], and analyzed colony formation. We found that this led to a slight but significant increase in colony number and size compared to the Ovol2-intact control (Fig. 5g). Interestingly, simultaneous deletion of Zeb1, an EMT-promoting factor that we previously shown to be an Ovol2 target in developing mammary epithelium [28], normalized this increase a

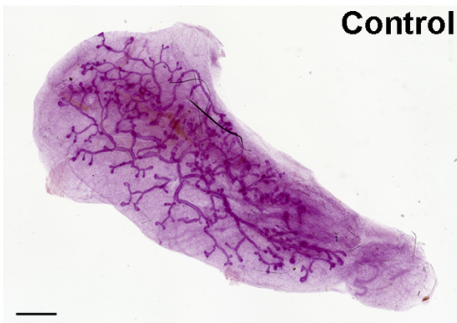

b
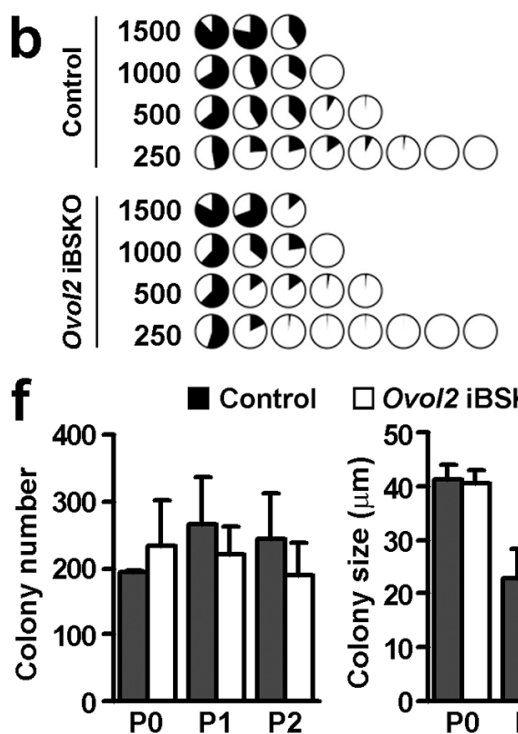

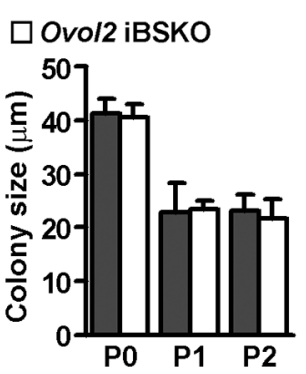

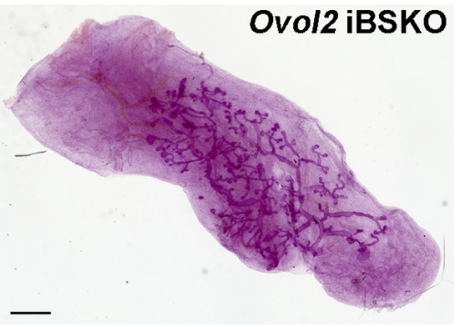
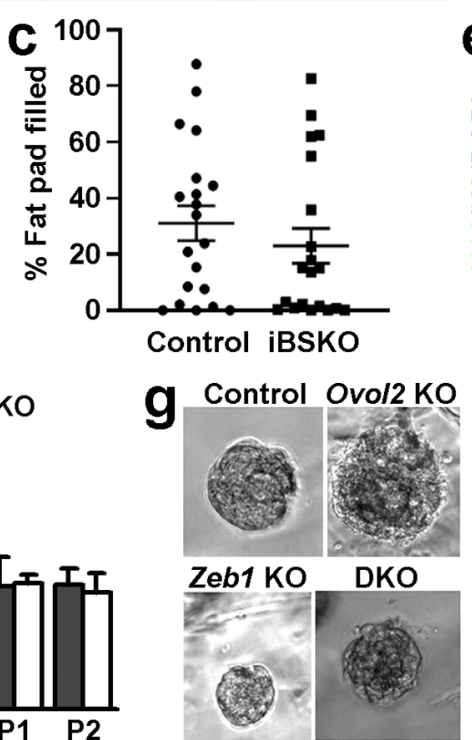
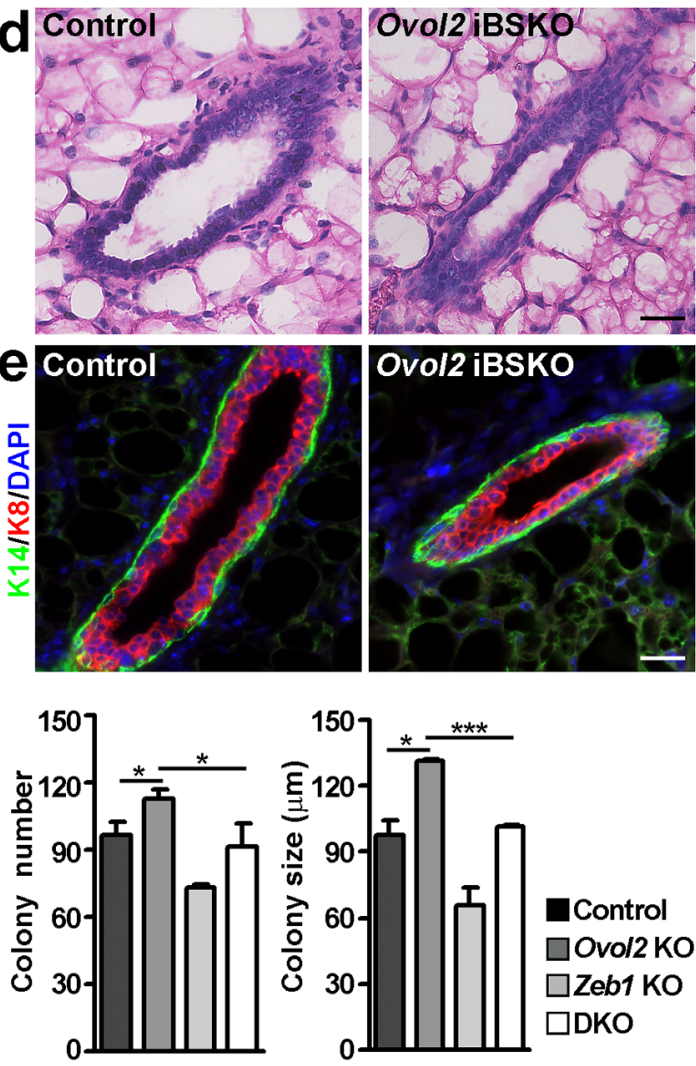

Fig. 5 Ovol2-deficient basal cells show largely normal regenerative potential and colony formation. a, Representative transplants derived from tdTomato $^{+}$basal cells isolated from control and Ovol2 iBSKO littermates at one month after tamoxifen injection. Scale bar: $1 \mathrm{~mm}$. b-c, Summary of limiting dilution transplantation results using 3 pairs of donor mice. Each pie diagram (b) represents an outgrowth, and data for percent of fat pad filled are summarized in (c). A tree that is $>5 \%$ of the fat pad indicates a positive take. Each experiment included 1 pair of Ovol2 iBSKO and control littermate as donor mice, and basal cells from each were transplanted at the indicated number onto the contralateral sides of the same host mice. d-e, H/E staining (d) and indirect immunofluoresence (e) of the transplants. Scale bar: $25 \mu \mathrm{m}$. f, Colony formation by control and Ovol2 iBKSO basal cells at one month post-tamoxifen injection. P0, initial plating. Colonies from $\mathrm{P} 0$ were harvested and equal numbers of control and iBKSO cells were subsequently passaged once (P1) or twice (P2). Shown are summary of 3 independent experiments. $\mathbf{g}$, Colony formation by control (Ade-Cre-infected Ovol2 ${ }^{+/ f} ; \mathrm{Zebl}^{+/ \mathrm{f}}$ ), Ovol2 single (Ade-Cre-infected $O v o l 2^{\mathrm{f} / \mathrm{f}} ; Z e b 1^{+/ \mathrm{f}}$ ), Zeb1 single (Ade-Cre-infected $O v o l 2^{+/ \mathrm{f}} ; Z e b 1^{\mathrm{f} / \mathrm{f}}$ ), and Ovol2/Zebl double (D; Ade-Cre-infected Ovol2 $\left.{ }^{\mathrm{f} / \mathrm{f}} ; \mathrm{Zebl}^{\mathrm{f} / \mathrm{f}}\right)$ knockout (KO) basal cells. $n=3$ for each. Represent colony images are shown on the left, and summary of three independent experiments using different mice are shown on the right. *** $p<0.005, * p<0.05$ 
(Fig. $5 \mathrm{~g}$ ). Taken these data together with our in vivo findings, we surmise that $O$ vol2's role in adult mammary basal cell proliferation/self-renewal is context-specific but centers around the inhibition of EMT-associated gene expression.

\section{Discussion}

The acquisition of stem-like traits by breast cancer cells through ectopic expression of EMT-inducing factors has led to the suggestion that EMT-like plasticity may also confer stemness to normal epithelial cells [20, 22-24]. However, lineage tracing experiments in vivo has revealed that mammary basal cells expressing elevated levels of EMT-associated genes (e.g., S100a4, Zeb1) actually show reduced regenerative potential than other basal cells [19]. Our work adds clarification to this issue of debate by showing that adult mammary basal cells with attenuated molecular epithelial traits and enhanced molecular mesenchymal traits, as a consequence of losing epithelial-promoting transcription factor Ovol2, exhibit similar in vivo transplantability as their Ovol2-expressing counterparts. Thus, certain alterations in basal cell EMP can be uncoupled from functional manifestation of stem cell self-renewal.

Interestingly, colony formation of Ovol2-deficient cells in vitro is variably affected depending on the exact method of deletion, with SMA-CreER-directed deletion in vivo leading to no change whereas Ade-Cre-directed deletion in vitro leading to a detectable increase. As such, Ovol2 shows a context-dependent requirement in adult basal cell function.

Our findings also provide new insights into the difference and similarity between developmental and adult mammary basal cells in their EMP tolerance. $\mathrm{K} 14$-Cre-driven panepithelial-deletion of $\mathrm{Ovol} 2$ almost completely arrests pubertal ductal morphogenesis, and FACS-sorted Ovol2-deficient basal cells from these mice cannot efficiently regenerate mammary trees upon transplantation [28]. In contrast, basal-specific Ovol2 deletion does not significantly compromise or enhance regenerative potential at least under the conditions of our experiments (this work). In both cases, however, Ovol2 loss results in detectable changes in the expression of EMT-associated genes, indicating a shared requirement by developmental and adult basal cells for Ovol2 to robustly maintain their transcriptional programs in a quasi-mesenchymal, but still epithelial, state.

How to explain the differential responses to altered EMP by the developmental vs. adult basal cells as well as by the adult basal cells in which Ovol2 is deleted using SMACreER or Ade-Cre? The extent and duration of EMT-like changes might be critical. The relatively slow turnover of adult basal cells during homeostasis, compared to their developmental counterparts during pubertal development, may implicate a smaller demand for EMP maintenance.
Functional perturbations to adult basal cells may manifest only after the cells are in an EMT-like state for more extensive periods of time. Unfortunately, we were unable to efficiently transplant basal cells from both WT and Ovol2-deficient mice that are five months of age (data not shown), preventing us from experimentally testing this possibility. The precise cell adhesion status of Ovol2-deficient basal cells may also influence basal cell proliferation/selfrenewal. As such, extensive experimental manipulation in culture may have altered cell adhesion to the degree that unmasks an advantageous effect of enhanced EMT-like features in adult basal cells. Lastly, we cannot fully exclude the possibility that the tdTomato-positive basal cell population still contains residual Ovol2-intact cells because of differential Cre-mediated recombination efficiency at different loci, and these cells may have also contributed to regeneration in vivo and colony formation in vitro.

Another important difference between the pan-epithelial Ovol2 knockout and basal cell-specific Ovol2 knockout models is that $O v o l 2$ is also deleted in luminal cells of the former. Loss of Ovol2 may compromise the function of luminal cells, which in turn influence basal cell function, explaining the severe defects in mammary epithelial development and regeneration in the pan-epithelial mutant mice. Of interest, organoids produced by terminal end buds isolated from pan-epithelial Ovol2 knockout mice contain almost entirely K14-positive (basal) cells but few K18-positive (luminal) cells (data not shown), suggesting a possible role for Ovol2 in promoting luminal differentiation. Whether this function occurs at the binary junction between basal and luminal cells or entirely in luminal cells will be an interesting future pursuit. Our observation that basal cell-specific deletion of Ovol2 does not affect basal/luminal cell frequencies seems to suggest the latter. This said, it is also possible that $S M A$ CreER, while proven by our work to be effective in directing gene deletion in bulk basal cells during adulthood, may not efficiently target the specific basal cell subsets that express low levels of Acta2 but are already destined to a luminal fate.

In summary, our work uncovers a functional regulator (Ovol2) of adult basal cell EMP, but at the same time underscores the ability of basal cells to tolerate molecular perturbations associated with EMT enhancement without drastically compromising their self-renewal potential. These findings may also inform our understanding of the mechanistic connection between EMT and stemness of basal-like cells in breast cancer.

\section{Methods}

\section{Mice}

SMA-CreER and $O v o l 2^{\mathrm{f} / \mathrm{f}}$ mice were as previously reported $[7,28]$. ROSA ${ }^{\text {tdTomato }}$ mice were purchased from the Jackson 
Laboratory (Stock \# 007909). Primers used for genotyping are listed in Table S1. All mouse experiments have been approved by and conform to the regulatory guidelines of the Institutional Animal Care and Use Committee of the University of California, Irvine.

\section{Tamoxifen Treatment}

Tamoxifen (Sigma, T5648) was prepared at a concentration of $20 \mathrm{mg} / \mathrm{ml}$ in corn oil (Sigma, C8267). Eight-week-old mice received two intraperitoneal (IP) injections $(24 \mathrm{~h}$ apart) of $2 \mathrm{mg}$ of tamoxifen per $20 \mathrm{~g}$ body weight or corn oil (100 $\mu \mathrm{l}$ per $20 \mathrm{~g}$ body weight). Mammary glands were harvested at $24 \mathrm{~h}$ to five months after the second injection.

\section{Isolation of Mammary Epithelial Cells}

Mammary cell preparations were performed as previously described [28, 30, 31]. Briefly, mammary glands were isolated from female mice and placed in the digestion mix [DMEM/ F12 (1:1) with 5\% fetal bovine serum (FBS) (Omega Scientific, FB-02), $300 \mathrm{U} / \mathrm{mL}$ collagenase (Sigma, C9891) and $100 \mathrm{U} / \mathrm{mL}$ hyaluronidase (Sigma, H3506)] for $1.5 \mathrm{~h}$ at $37^{\circ} \mathrm{C}$. Cells were pelleted and resuspended in red blood cell lysis buffer (Sigma, R7757). Single cell suspension was obtained by further treatment with $0.25 \%$ trypsin-EDTA (Gibco, 25200), $10 \mathrm{mg} / \mathrm{mL}$ DNase (Sigma, DN25), and $5 \mathrm{mg} / \mathrm{mL}$ dispase (Stem Cell Technologies, 07913), followed by filtration using a $40 \mu \mathrm{m}$-pore mesh filter (SWiSH, TC70-MT-1).

\section{Cell Labeling and Flow Cytometry}

Single cell suspension from above was stained using the following antibodies and reagents: anti-CD49f-FITC (1:250, Bio Legend, 102205), anti-EpCAM-PE-Cy7 (1:250, Bio Legend, 118215), anti-lineage-APC [1:250; including APC-CD45 (BD Biosciences, 559864), APC-CD31 (BD Biosciences, 551262), APC-TER119 (BD Biosciences, 557909)], and SytoxBlue (Invitrogen, S3457). Flow cytometry analysis and sorting were performed on a Novocyte and FACSAria (Becton Dickenson UK), respectively.

\section{RT-qPCR Analysis}

Total RNA was extracted from FACS-sorted cells using RNeasy Mini Kit with on-column DNase treatment according to manufacturer's protocol (Zymo Research, R1050). cDNA was synthesized using High-Capacity cDNA Reverse Transcription Kit (Thermo Fisher Scientific, 4368814) according to manufacturer's instructions. Real-time PCR was performed using a SYBR Green Supermix (BioRad, 1725122) on a CFX96 RT-qPCR system, and data were analyzed using the $2-\Delta \Delta \mathrm{CT}$ method. Primers used for qPCR are listed in Table S1.

\section{Immunohistochemistry and Mammary Gland Whole-mount Analysis}

Immunohistochemistry was performed as previously described [28, 30, 31] using the following antibodies: vimentin (rabbit, Cell Signaling, 5741P), K14 (chicken or rabbit, a gift from J. Segre, National Institutes of Health, Bethesda, MD) and K8 (rat, Developmental Studies Hybridoma Bank).

For whole-mount analysis, the \#4 pair of mammary glands were dissected and fixed in Carnoy's fixative (10\% acetic acid, $30 \% \mathrm{CHCl}_{3}, 60 \%$ ethanol) for $2-4 \mathrm{~h}$. Fixed tissues were treated with a gradient of ethanol $(100 \%, 70 \%, 30 \%)$ and then washed with sterile water for $10 \mathrm{~min}$. Tissues were then incubated with carmine-alum staining solution as previously described. Images were captured using a Keyence microscope.

\section{Cleared Fat Pad Transplantation}

Fat pad clearing and transplantation was as previously described [28, 30, 31]. Briefly, single cell suspensions of sorted tdTomato-positive basal cells from Ovol2 iBSKO and control littermates were prepared as described above and diluted in a 1:1 solution of $5 \%$ FBS media/Matrigel at limiting dilutions $(2,000,1,500,1,000,500$, and 250 cells per $10 \mu \mathrm{l})$. Ten $\mu \mathrm{L}$ of the cell/Matrigel solution was injected into cleared fat pads of \#4 mammary glands of 3-week-old C57BL/6 females, with each host mouse receiving contralateral injections of $\mathrm{Ovol2}$ iBSKO and control samples. Outgrowths were analyzed 8-9 weeks later using whole-mount carmine alum staining.

For histology and immunostaining of carmine-stained mammary outgrowths, mammary whole-mounts were immersed in xylene to remove excess permount and processed in paraffin overnight before embedding. The embedded outgrowths were then sectioned, and stained with H/E or K8/K14 antibodies using established methods [32, 33].

\section{D-matrigel Colony Assay and Ade-Cre Infection}

FACS-sorted tdTomato-positive basal cells from Ovol2 iBSKO and control littermates were resuspended in a chilled 1:1 solution of EpiCult-B medium (Stem Cell Technologies, 05610)/growth factor-reduced Matrigel (BD Biosciences, CB-40230), and 4000 cells were plated into one well from 8 -well chamber slides (Thermo Fisher Scientific, 154534). After Matrigel hardens, $400 \mu \mathrm{l}$ of EpiCult-B medium containing $10 \mathrm{ng} / \mathrm{mL}$ EGF (Millipore, 01-107), $10 \mathrm{ng} / \mathrm{mL}$ bFGF (PeproTech, 100-18B), and $4 \mu \mathrm{g} / \mathrm{mL}$ heparin (Stem Cell Technologies, 07980) was added into each well. Culture 
medium was changed every three days for two weeks, followed by counting colony number and measuring colony size. To passage the colonies, medium was first removed and Matrigel dissolved using $200 \mu \mathrm{L}$ of dispase for at least $20 \mathrm{~min}$. Single cells were obtained by incubation in $0.25 \%$ Trypsin-EDTA followed by filtration using a $40 \mu \mathrm{m}$ filter. Cells were then re-plated as described above.

GFP-expressing Ade-Cre viruses were purchased from Vector Biolabs. FACS-sorted basal cells were plated at 10 million/mL/well in 24-well plates in DMEM/F12 (Stemcell Technologies, 36254) containing 2\% FBS, 10 mM HEPES (Millipore Sigma, H3375), $10 \mathrm{ng} / \mathrm{ml}$ EGF (Invitrogen, PMG-8043), $250 \mathrm{ng} / \mathrm{ml}$ Rspo1 (R\&D, 3474-RS), $100 \mathrm{ng} /$ $\mathrm{ml}$ Noggin (Fisher Scientific, 50-399-006), and $10 \mu \mathrm{M}$ Rock inhibitor Y27632 (Millipore Sigma, SCM075), and cultured overnight. Cells were then infected with Ade-Cre at a multiplicity of infection (MOI) of 10 for overnight in 24-well ultra-low attachment surface polystyrene plate (Corning, REF 3473). Cells were harvested the next day, and treated with $400 \mu \mathrm{L}$ TrypLE Select (GIBCO, 12605-010) for $20 \mathrm{~min}$ at $37^{\circ} \mathrm{C}$ followed by neutralization with $2 \mathrm{~mL}$ HBSS buffer (GIBCO, 14025134). $\mathrm{GFP}^{+}$cells were FACS-sorted for colony formation assay.

\section{Statistics}

Experiments were performed on at least 3 biological replicates or repeated at least twice. The sample size and number of independent experiments are indicated in the relevant figure legends. For analysis of differences between groups, Student's unpaired $t$-test was performed with 2-tailed in Excel. $p$ values of 0.05 or less were considered statistically significant. Error bars represent mean \pm SEM.

Supplementary Information The online version contains supplementary material available at https://doi.org/10.1007/s10911-021-09508-0.

Acknowledgements We thank the Institute for Immunology FACS Core Facility at the University of California, Irvine for expert service. This work was supported by NIH Grants R01-GM123731 and R01-AR068074 (X.D.).

Authors Contributions X.D. conceived the study and directed the project. P. S. and Y. H. performed the experiments and data analysis. M.P. contributed the SMA-CreER mice. X.D. and P.S. wrote the manuscript.

\section{Declarations}

Competing Interests The authors declare that they have no competing interests.

Open Access This article is licensed under a Creative Commons Attribution 4.0 International License, which permits use, sharing, adaptation, distribution and reproduction in any medium or format, as long as you give appropriate credit to the original author(s) and the source, provide a link to the Creative Commons licence, and indicate if changes were made. The images or other third party material in this article are included in the article's Creative Commons licence, unless indicated otherwise in a credit line to the material. If material is not included in the article's Creative Commons licence and your intended use is not permitted by statutory regulation or exceeds the permitted use, you will need to obtain permission directly from the copyright holder. To view a copy of this licence, visit http://creativecommons.org/licenses/by/4.0/.

\section{References}

1. Inman JL, Robertson C, Mott JD, Bissell MJ. Mammary gland development: Cell fate specification, stem cells and the microenvironment. Dev. 2015

2. Watson CJ, Khaled WT. Mammary development in the embryo and adult: new insights into the journey of morphogenesis and commitment. Development. 2020

3. Fu NY, Nolan E, Lindeman GJ, Visvader JE. Stem cells and the differentiation hierarchy in mammary gland development. Physiol Rev. 2020.

4. Van Amerongen R, Bowman AN, Nusse R. Developmental stage and time dictate the fate of $\mathrm{Wnt} / \beta$-catenin- responsive stem cells in the mammary gland. Cell Stem Cell. 2012.

5. Deugnier MA, Teulière J, Faraldo MM, Thiery JP, Glukhova MA. The importance of being a myoepithelial cell. Breast Cancer Res. 2002.

6. Van Keymeulen A, Rocha AS, Ousset M, Beck B, Bouvencourt G, Rock J, Sharma N, Dekoninck S, Blanpain C. Distinct stem cells contribute to mammary gland development and maintenance. Nature. 2011.

7. Prater MD, Petit V, Russell IA, Giraddi RR, Shehata M, Menon S, Schulte R, Kalajzic I, Rath N, Olson MF, et al. Mammary stem cells have myoepithelial cell properties. Nat. Cell Biol. 2014.

8. Rios AC, Fu NY, Lindeman GJ, Visvader JE. In situ identification of bipotent stem cells in the mammary gland. Nature. 2014.

9. Shackleton M, Vaillant F, Simpson KJ, Stingl J, Smyth GK, AsselinLabat ML, Wu L, Lindeman GJ, Visvader JE. Generation of a functional mammary gland from a single stem cell. Nature. 2006.

10. Stingl J, Eirew P, Ricketson I, Shackleton M, Vaillant F, Choi D, Li HI, Eaves CJ. Purification and unique properties of mammary epithelial stem cells. Nature. 2006.

11. Haensel D, Dai X. Epithelial-to-mesenchymal transition in cutaneous wound healing: where we are and where we are heading. Dev Dyn. 2018;247:473-80.

12. Nieto MA, Huang RYYJ, Jackson RAA, Thiery JPP. EMT: 2016. Cell. 2016.

13. Pastushenko I, Blanpain C. EMT transition states during tumor progression and metastasis. Trends Cell Biol. 2019.

14. Sha Y, Haensel D, Gutierrez G, Du H, Dai X, Nie Q. Intermediate cell states in epithelial-to-mesenchymal transition. Phys Biol. 2019.

15. Thiery JP, Acloque H, Huang RYJ, Nieto MA. Epithelial-mesenchymal transitions in development and disease. Cell. 2009.

16. Yang J, Antin P, Berx G, Blanpain C, Brabletz T, Bronner M, Campbell K, Cano A, Casanova J, Christofori G, et al. Guidelines and definitions for research on epithelial-mesenchymal transition. Nat Rev Mol Cell Biol. 2020.

17. Lim E, Wu D, Pal B, Bouras T, Asselin-Labat ML, Vaillant F, Yagita H, Lindeman GJ, Smyth GK, Visvader JE. Transcriptome analyses of mouse and human mammary cell subpopulations reveal multiple conserved genes and pathways. Breast Cancer Res. 2010. 
18. Nassour M, Idoux-Gillet Y, Selmi A, Côme C, Faraldo MLM, Deugnier MA, Savagner P. Slug controls stem/progenitor cell growth dynamics during mammary gland morphogenesis. PLoS One. 2012.

19. Sikandar SS, Kuo AH, Kalisky T, Cai S, Zabala M, Hsieh RW, Lobo NA, Scheeren FA, Sim S, Qian D, et al. Role of epithelial to mesenchymal transition associated genes in mammary gland regeneration and breast tumorigenesis. Nat Commun. 2017.

20. Ye X, Tam WL, Shibue T, Kaygusuz Y, Reinhardt F, Ng Eaton E, Weinberg RA. Distinct EMT programs control normal mammary stem cells and tumour-initiating cells. Nature. 2015.

21. Chaffer CL, Marjanovic ND, Lee T, Bell G, Kleer CG, Reinhardt F, D'Alessio AC, Young RA, Weinberg RA. XPoised chromatin at the ZEB1 promoter enables breast cancer cell plasticity and enhances tumorigenicity. Cell. 2013.

22. Guo W, Keckesova Z, Donaher JL, Shibue T, Tischler V, Reinhardt F, Itzkovitz S, Noske A, Zürrer-Härdi U, Bell G, et al. Slug and Sox 9 cooperatively determine the mammary stem cell state. Cell. 2012.

23. Mani SA, Guo W, Liao MJ, Eaton EN, Ayyanan A, Zhou AY, Brooks M, Reinhard F, Zhang CC, Shipitsin M, et al. The epithelial-mesenchymal transition generates cells with properties of stem cells. Cell. 2008.

24. Morel AP, Lièvre M, Thomas C, Hinkal G, Ansieau S, Puisieux A. Generation of breast cancer stem cells through epithelialmesenchymal transition. PLoS One. 2008.

25. Stemmler MP, Eccles RL, Brabletz S, Brabletz T. Non-redundant functions of EMT transcription factors. Nat Cell Biol. 2019.

26. Yang J, Mani SA, Donaher JL, Ramaswamy S, Itzykson RA, Come C, Savagner P, Gitelman I, Richardson A, Weinberg RA. Twist, a master regulator of morphogenesis, plays an essential role in tumor metastasis. Cell. 2004.
27. Hong T, Watanabe K, Ta CH, Villarreal-Ponce A, Nie Q, Dai X. An Ovol2-Zeb1 mutual inhibitory circuit governs bidirectional and multi-step transition between epithelial and mesenchymal states. PLoS Comput Biol. 2015;11.

28. Watanabe K, Villarreal-Ponce A, Sun P, Salmans ML, Fallahi $\mathrm{M}$, Andersen B, Dai X. Mammary morphogenesis and regeneration require the inhibition of EMT at terminal end buds by ovol2 transcriptional repressor. Dev Cell. 2014;29.

29. Han Y, Villarreal-Ponce A, Gutierrez Jr. G, Nguyen Q, Sun P, Wu T, Sui B, Berx B, Brabletz T, Kessenbrock K, Zeng YA, Watanabe $\mathrm{K}$, Dai X. Coordinate control of basal epithelial cell fate and stem cell maintenance by core EMT transcription factor Zeb1. Cell Reports. In press. 2022.

30. Gu B, Sun P, Yuan Y, Moraes RC, Li A, Teng A, Agrawal A, Rhéaume C, Bilanchone V, Veltmaat JM, et al. Pygo2 expands mammary progenitor cells by facilitating histone H3 K4 methylation. J Cell Biol. 2009;185:811-26.

31. Gu B, Watanabe K, Sun P, Fallahi M, Dai X. Chromatin effector Pygo 2 mediates wnt-notch crosstalk to suppress luminal/alveolar potential of mammary stem and basal cells. Cell Stem Cell. 2013;13:48-61.

32. Sun P, Yuan Y, Li A, Li B, Dai X. Cytokeratin expression during mouse embryonic and early postnatal mammary gland development. Histochem Cell Biol. 2010;133.

33. Watanabe K, Villarreal-Ponce A, Sun P, Salmans ML, Fallahi M, Andersen B, Dai X. Mammary morphogenesis and regeneration require the inhibition of EMT at terminal end buds by ovol2 transcriptional repressor. Dev Cell. 2014;29:59-74.

Publisher's Note Springer Nature remains neutral with regard to jurisdictional claims in published maps and institutional affiliations. 